\title{
The effect of royal jelly on the growth of breast cancer in mice
}

\author{
SHUANG ZHANG ${ }^{1,2}$, QIQI SHAO ${ }^{1}$, HAIYANG GENG ${ }^{1}$ and SONGKUN SU ${ }^{1}$ \\ Colleges of ${ }^{1}$ Bee Science and ${ }^{2}$ Life Science, Fujian Agriculture and Forestry University, \\ Fuzhou, Fujian 350002, P.R. China
}

Received January 4, 2016; Accepted May 25, 2017

DOI: $10.3892 / \mathrm{ol} .2017 .7078$

\begin{abstract}
Due to various pharmacological properties, including antioxidative, anti-inflammatory and antibiotic properties, royal jelly (RJ) has been widely consumed in daily diets in numerous countries. In the present study, the effect of RJ on 4T1-bearing mice was investigated. The study was performed by feeding 4T1-bearing mice with RJ using either the prophylactic-therapeutic (PTRJ) or therapeutic (TRJ) method. The experimental results for the PTRJ group demonstrated that the weight of tumor was significantly reduced (RJ 0.5 and $1.5 \mathrm{~g} / \mathrm{kg}$ ); and in the serum, the levels of interleukin (IL)-2 (RJ 0.5 and $1.5 \mathrm{~g} / \mathrm{kg}$ ), interferon (IFN)- $\alpha$, superoxide dismutase (SOD) and total antioxidant capacity (T-AOC) were significantly elevated, but the concentrations of IL-4 (RJ 0.5 and $1.5 \mathrm{~g} / \mathrm{kg}$ ) and IL-10 (RJ $1.0 \mathrm{~g} / \mathrm{kg}$ ) were significantly decreased. In addition, the activities of T-AOC and glutathione reductase (GR) were significantly improved in the liver, whereas in the kidney, the activities of T-AOC and GR were significantly increased only under the dose of $0.5 \mathrm{~g} / \mathrm{kg}$. For the TRJ group, the antitumor effect of RJ was not significant; the change in IL-2, IFN- $\alpha$, SOD and T-AOC levels in the serum, and the change in T-AOC and GR in liver were similar to those observed in the PTRJ groups. RJ treatment was demonstrated to reduce the development of breast tumor in mice, and simultaneously improve the antioxidant capacity of the serum, liver and kidney, particularly using the prophylactic-therapeutic method. These results corroborated the efficacy of RJ supplementation in diets. The results of the present study suggest that the antioxidant and immunomodulatory activities of RJ serve an important role on antitumor growth.
\end{abstract}

\section{Introduction}

Royal jelly $(\mathrm{RJ})$ is a thick and milky liquid secreted by hypopharyngeal, and mandibular glands of young worker bees (Apismellifera L.), and is used to feed the larvae (1). RJ exhibits

Correspondence to: Professor Songkun Su, College of Bee Science, Fujian Agriculture and Forestry University, 15 Shangxiadian Road, Jianxin, Cangshan, Fuzhou, Fujian 350002, P.R. China

E-mail: susongkun@zju.edu.cn

Key words: royal jelly, breast cancer, antioxidant, immunomodulatory a wide variety of functional properties, and has been widely used in cosmetics, healthy foods and commercial medical products in numerous countries. Additionally, RJ possesses various properties, including antimicrobial (2), anti-inflammatory $(3,4)$, hepatoprotective $(5)$, antisarcopenia (6), insulin-like action (7) and antihypercholesterolemic (8) properties. In the recent decades, there have been an increasing number of studies investigating the effects of RJ.

Regarding breast cancer, RJ has been reported to be able to inhibit the growth-promoting effect of bisphenol A, which is an estrogen that enhances the proliferation of MCF-7 mammary cancer cells (9). RJP30, a substance obtained from RJ crude protein, which was extracted by precipitation with $30 \%$ ammonium sulfate, was demonstrated to be cytotoxic for HeLa human cervicouterine carcinoma cells, and diminish cell density to $50 \%$ of the original carcinoma cell after 7 days of treatment (10). RJ exhibited no significant effects on the formation of metastases in spontaneous mammary carcinoma of CBA mice when it was given intraperitoneally or subcutaneously (11). However, when $\mathrm{RJ}$ and tumor cells were injected synchronously into mice, RJ was able to inhibit the formation of metastases in the lung (11). The therapeutic properties of RJ have been examined in various cells, including MCF-7 (mouse macrophages), spontaneous mammary carcinoma cells and methylcholanthrene-induced fibrosarcoma CBA mouse cells $(9,11,12)$.

RJ exhibits estrogenic activity. It was identified that RJ stimulated the expression of endogenous estrogen-responsive genes [presenilin 2 and vascular endothelial growth factor (VEGF)] by activating estrogen receptors in MCF-7 cells, and RJ could restore the expression of VEGF in uterus of rats that received bilateral ovariectomy (13). Experimental results demonstrated that four compounds from RJ can improve the transcription of a reporter gene, which contained an estrogen-responsive element, and subcutaneously injecting immature rats with those compounds for 23 days induced mild hypertrophy in the luminal epithelium of the rat uterus (14). With evidence that it was an etiological cause of breast cancer, estrogen attracted numerous studies on its association with breast carcinogenesis (15). Samavat and Kurzer (16) evaluated the roles and metabolites of circulating, and urinary estrogens in human breast cancer. Endocrine therapy is regarded as one of the earliest molecular-targeting therapies applied in breast cancer treatment (17). However, the effect of RJ on breast cancer has been seldom studied.

The 4T1 tumor closely mimics human breast cancer with regards to anatomically correct site, immunogenicity and 
growth characteristics $(18,19)$. Therefore, the $4 \mathrm{~T} 1$ tumor is a suitable model for testing the therapy effects under experimental conditions.

Breast cancer is associated with high morbidity and mortality rates among women in developed and developing countries (20). However, the effect of RJ on mammary cancer cells remains unclear. Therefore, a detailed and comprehensive study on the effect of RJ on breast cancer may aid in understanding the association between $\mathrm{RJ}$ and breast cancer.

The present study aimed to analyze the effect of RJ on breast cancer. The primary purpose of the current study was to investigate the potential antioxidant ability and immune response of $\mathrm{RJ}$ on 4T1-bearing BALB/c mice, and changes in the weights of different organs.

\section{Materials and methods}

Cell culture. Mouse mammary tumor cells (4T1) were purchased from Culture Collection of the Chinese Academy of Sciences (Shanghai, China). All culture work was performed under strict aseptic conditions. Cells were cultured in RPMI-1640 (HyClone; GE Healthcare Life Sciences, Logan, UT, USA) supplemented with $10 \%$ fetal bovine serum (Gibco; Thermo Fisher Scientific, Inc., Waltham, MA, USA) at $37^{\circ} \mathrm{C}$ in a $5 \% \mathrm{CO}_{2}$ humidified incubator.

Mice. Female BALB/c mice (4 weeks old, 18-22 g) were purchased from Shanghai SLAC Laboratory Animal Co., Ltd. (Shanghai, China), and the present study was ethically approved by the Animal Care and Use Committee, Laboratory Animal Center of Fujian Medical University (Fuzhou, China). The mice were housed four to a cage under specific pathogen-free conditions at constant temperature $\left(25-28^{\circ} \mathrm{C}\right)$ with a $12 \mathrm{~h}$ light/12 h dark schedule, and were fed with autoclaved food and water.

Tumor model. All 4T1-bearing mice were injected subcutaneously with 4T1 cells $\left(4 \times 10^{5}\right)$ suspended in $0.2 \mathrm{ml}$ of PBS in the mammary fat pads (21) in the right flank of each mouse when treated for 14 days. The tumor was detected through palpation around the induction area.

$R J$ and treatment regimen. Fresh RJ was obtained from a local beekeeping association, Yuhang Apiary (Zhejiang, China), and was stored at $-20^{\circ} \mathrm{C}$ before prior to use. A total of 56 female $\mathrm{BALB} / \mathrm{c}$ mice were divided into seven groups with eight mice in each group. One of the seven groups was a 4T1 control group, and was fed with water with no RJ content during the experiment. Three groups were administered orally with RJ solution using a prophylactic-therapeutic method (PTRJ groups): The mice were treated with RJ 14 days prior to the transplantations of tumor cells, and then continued to be treated with RJ for 28 consecutive days. The other three groups were administered with RJ using a therapeutic method (TRJ groups): after the transplantations of tumor cells, the mice were treated with RJ for 28 days. For each of the three groups receiving RJ treatment, the daily doses of RJ were $0.5,1.0$ and $1.5 \mathrm{~g} / \mathrm{kg}$, respectively. RJ solution was prepared by dissolving RJ in distilled water (22). The weight of the mice and tumor sizes were measured periodically. All the mice were sacrificed after $24 \mathrm{~h}$ of the final administration of
RJ (22), subsequently blood and tissue samples were collected, and stored for further experiments.

Weight of organs. Following the sacrifice of the mice, necropsy was performed by removing the tumor, kidney, thymus, spleen, lung and liver from the bodies. Then, these organs were weighed with using a one over ten-thousand analytical balance.

Antioxidant and immunomodulatory activities of serum. The blood samples were centrifuged for $10 \mathrm{~min}$ at 3,000 $\mathrm{x} g$ at room temperature, and the serum was aliquoted and stored in plastic eppendorf tubes. The serum was analyzed for the concentrations of various cytokines, including interleukin (IL)-2, IL-4, IL-10, interferon (IFN)- $\alpha$ and IFN- $\gamma$, all of which are associated with immunomodulation, using Mouse IL-2 Quantikine ELISA kit (cat no. M2000), Mouse IL-4 Quantikine ELISA kit (cat no. M4000B), Mouse IL-10 Quantikine ELISA kit (cat no. M1000B), Mouse IFN- $\alpha$ Quantikine ELISA kit (cat no. 42120-1) and Mouse IFN- $\gamma$ Quantikine ELISA kit (cat no. MIF00), according to the manufacturer's protocol, respectively. These ELISA kits were purchased from R\&D Systems, Inc., Minneapolis, MN, USA. The Total Antioxidant Capacity Assay kit (cat no. S0119) and Glutathione Reductase Assay kit (cat no. S0055) were purchased from Beyotime Institute of Biotechnology (Haimen, China) and were used to determine the concentrations of various antioxidant-associated indicators, including total antioxidant capacity (T-AOC) and glutathione reductase (GR), according to the manufacturer's protocol.

Antioxidant ability of liver and kidney. The effects of RJ on antioxidant markers were assessed by measuring the activities of liver- or kidney-associated enzymes, including superoxide dismutase (SOD), GR and T-AOC, in the homogenate using the following assay kits: Total Antioxidant Capacity Assay kit (cat no. S0119), Glutathione Reductase Assay kit (cat no. S0055) and Total Superoxide Dismutase Assay kit (cat no. S0102), according to the manufacturer's instructions. These assay kits were obtained from Beyotime Institute of Biotechnology.

Statistical analysis. All data are expressed as the mean \pm standard error of the mean for in vitro studies. Statistical analysis was performed using one-way analysis of variance followed with Tukey's post hoc test using the SPSS version 17 (SPSS, Inc., Chicago, IL, USA). $\mathrm{P}<0.05$ was considered to indicate a statistically significant difference.

\section{Results}

Effects of RJ on the weight of organs. As demonstrated in Fig. 1, PTRJ0.5 and PTRJ1.5 significantly inhibited tumor growth of 4T1 mammary tumors in female BALB/c mice as compared with the 4T1 control, but no significant antitumor effects were observed in the PTRJ1.0 group. In the TRJ groups, although the development of tumors was also inhibited, the inhibitory effect was not significant compared with the 4T1 control group. No significant differences were identified among the 4T1 control group and RJ groups regarding the mean weights of the lung, and kidney of the mice.

Compared with those in the 4T1 control group, the weight of the thymus and spleen in all three PTRJ groups was 

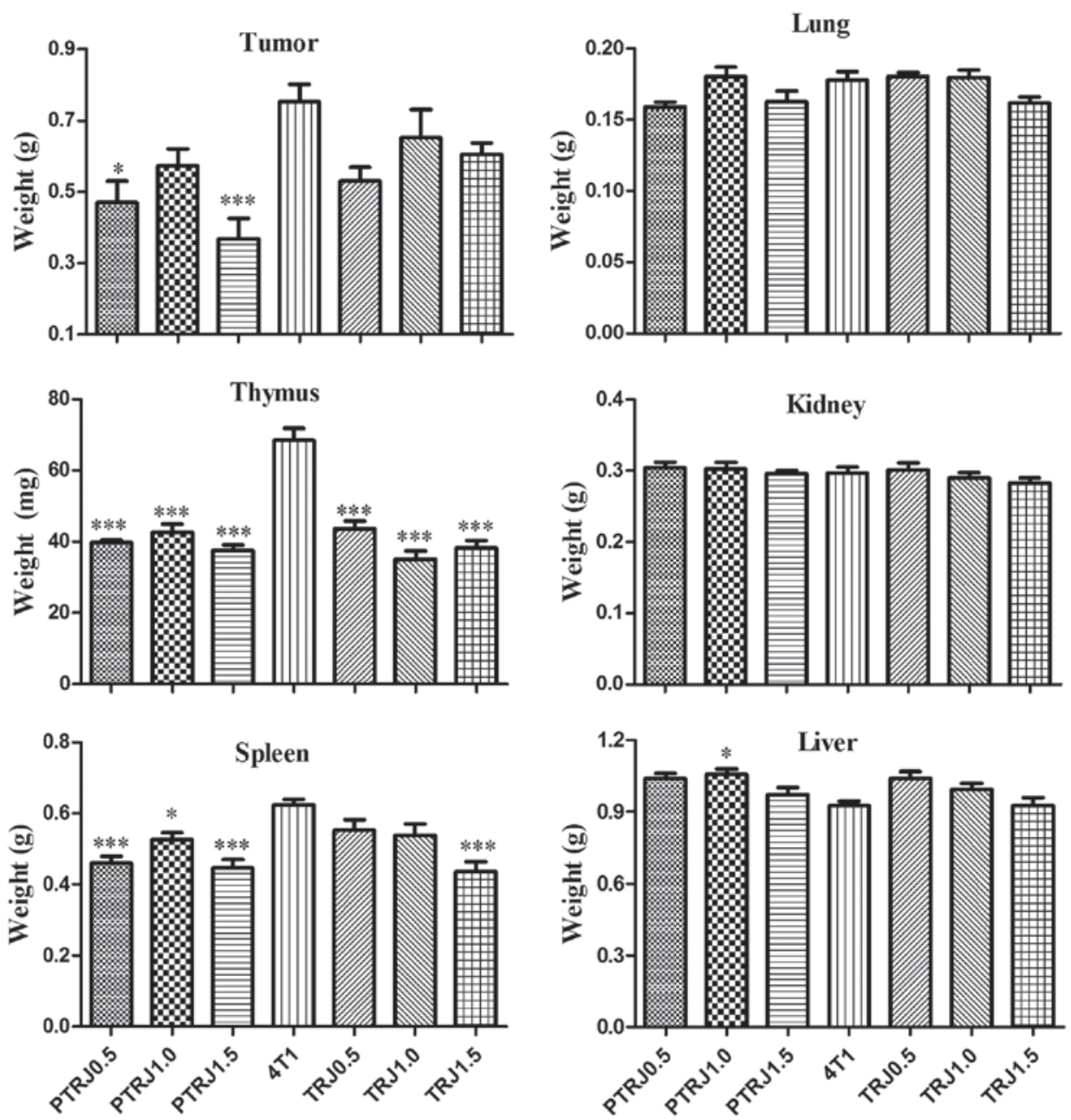

Figure 1. Mean weight of various organs, tumor, lung, spleen, liver, thymus and kidney, in the PTRJ, 4T1, and TRJ groups. Data are expressed as the mean \pm standard error of the mean. ${ }^{*} \mathrm{P}<0.05$ and ${ }^{* * *} \mathrm{P}<0.001$ vs. 4T1-bearing mice. PTRJ, prophylactic-therapeutic royal jelly group; TRJ, therapeutic royal jelly group.

significantly decreased. For the TRJ groups, the weight of thymus was only significantly reduced in the TRJ1.5 group compared with that of 4T1 control group. Contradictory results were identified regarding the weight of liver, all the TRJ and PTRJ groups possessed livers that were slightly heavier compared with that of $4 \mathrm{~T} 1$ control group, and was significantly heavier in the PTRJ1.0 group.

Effects of RJ on the serum. Fig. 2 presents the cytokine concentrations of the serum collected from mice. Compared with the 4T1 control group, PTRJ0.5, PTRJ1.5, TRJ0.5 and TRJ1.5 groups possessed significantly higher IL-2 concentrations. However, PTRJ0.5, PTR1.0 and TRJ1.5 groups had significantly lower levels of IL- 4 . The concentration of IFN- $\alpha$ in all RJ-treated groups was significantly higher compared with that in 4T1 control group. The level of IL-10 was significantly lower in the PTRJ1.0 group compared with that in 4T1 control group. IFN- $\gamma$ activity of all RJ-treated groups had similar values with that of 4T1 control group.

The activities of enzymatic antioxidants, including SOD and GR in the serum are presented in Fig. 3. When compared with the 4T1 control groups, SOD and T-AOC concentrations were significantly augmented in all RJ-treated groups.
Effects of RJ on the antioxidant ability of the liver and kidney. In the liver homogenates of RJ-treated groups, T-AOC and GR concentrations were significantly augmented compared with those of the 4T1 control group; while only TRJ1.5 group had an SOD value significantly higher compared with that of the 4T1 control group (Fig. 4). For the kidney homogenates, there was no significant difference in SOD activity among the groups in the experiment. The value of T-AOC and GR of all the groups was similar to those of the 4T1 control group, except the PTRJ0.5 group, which had significantly higher T-AOC and GR values.

\section{Discussion}

Previous studies have reported antitumoral effects of RJ on various types of tumor, including Ehrlich ascetic tumor (22), Sarcoma-180 ascites (23), and fibrosarcoma transplantable tumors (24). For the majority of tumors, RJ significantly prolongs the average life span of murine tumor models and the effect depends on the treatment protocol used $(22,23)$. The results of the present study suggested that the doses of RJ solution applied were nontoxic to the mice, as no abnormal fluctuation in weight or behaviors of mice was observed. 

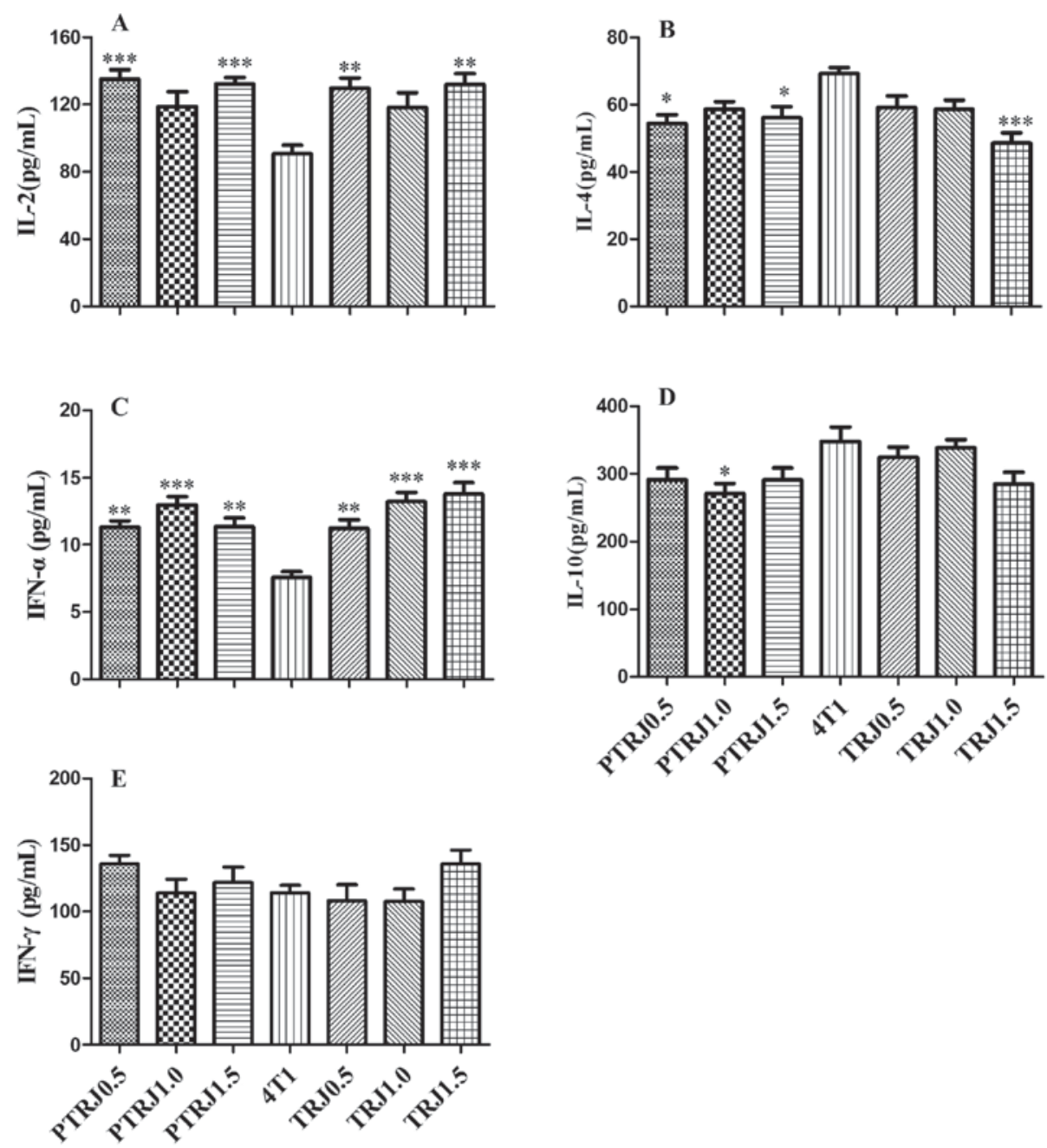

Figure 2. Mean levels of (A) IL-2, (B) IL-4, (C) IFN- $\alpha$, (D) IL-10 and (E) IFN- $\gamma$ cytokines in the serum of the PTRJ, 4 T1 and TRJ groups. Data are expressed as the mean \pm standard error of the mean. ${ }^{*} \mathrm{P}<0.05,{ }^{* *} \mathrm{P}<0.01$ and ${ }^{* * * *} \mathrm{P}<0.001$ vs. 4T1-bearing mice. PTRJ, prophylactic-therapeutic royal jelly group; TRJ, therapeutic royal jelly group; IFN, interferon; IL, interleukin.
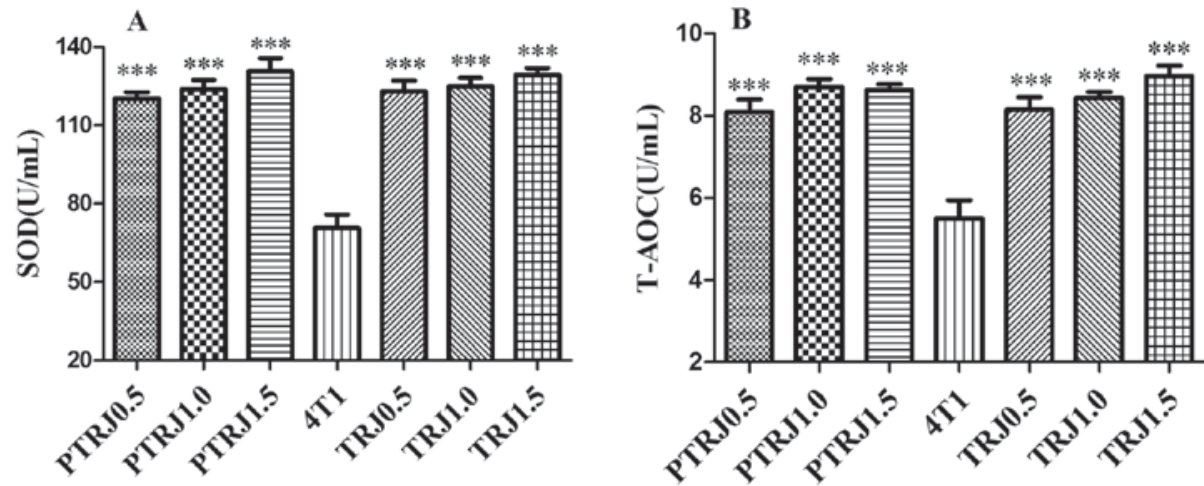

Figure 3. Antioxidant (A) SOD and (B) T-AOC levels in the serum of the PTRJ, 4T1 and TRJ groups. Data are expressed as the mean \pm standard error of the mean. ${ }^{* * *} \mathrm{P}<0.001$ vs. 4T1-bearing mice. PTRJ, prophylactic-therapeutic royal jelly group; TRJ, therapeutic royal jelly group; SOD, superoxide dismutase; T-AOC, total antioxidant capacity.

It was demonstrated that RJ significantly inhibited tumor growth in 4T1-bearing mice when orally administered in prophylactic-therapeutic method with the doses of 0.5 and $1.5 \mathrm{~g} / \mathrm{kg}$; however, RJ had no significant effect on growth of 4T1-bearing mice when given to mice following tumor cell inoculation. This result suggests that the antitumor effect of RJ primarily depends on the time of application, and RJ has little or no estrogen agonism on 4T1-bearing mice. Furthermore, the results demonstrated that administering RJ orally using the prophylactic-therapeutic method may benefit controlling 

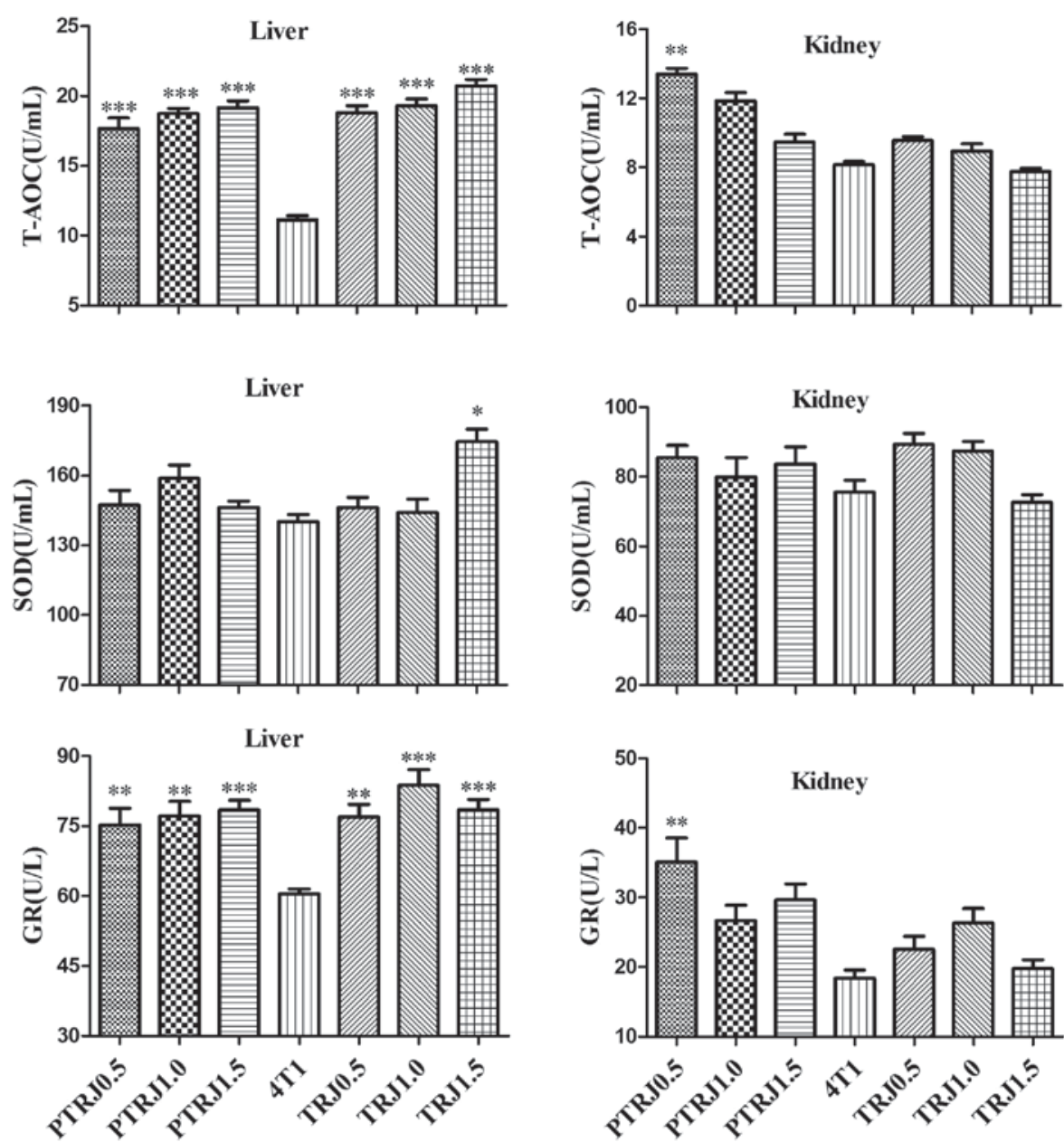

Figure 4. Antioxidant levels in the liver and kidney tissue of the PTRJ, 4T1, and TRJ groups. Data are expressed as the mean \pm standard error of the mean. ${ }^{*} \mathrm{P}<0.05,{ }^{* * *} \mathrm{P}<0.01$ and ${ }^{* * *} \mathrm{P}<0.001$ vs. 4T1-bearing mice. PTRJ, prophylactic-therapeutic royal jelly group; TRJ, therapeutic royal jelly group; SOD, superoxide dismutase; T-AOC, total antioxidant capacity; GR, glutathione reductase.

tumor growth. Accumulating evidence suggested the antitumor effect of RJ may be due to 10-hydroxy-2-decenoic acid and saturated fatty acids $(25,26)$.

No significant effects were observed on the weight of lung and kidney following both RJ treatments. Male (27) reported that cell-mediated and humoral immunity were affected during immune suppression, and this phenomenon was reflected in the changes in the functions of spleen and thymus. The thymus weight of all the groups, which were treated with RJ, was significantly reduced compared with that of 4T1 control group. In addition, all three PTRJ groups and the TRJ1.5 group exhibited significantly reduced spleen weights compared with the 4T1 control group. These results suggest that the weight of the spleen and thymus are significantly reduced with RJ treatment. Since the immune organ index is only a superficial indicator of immune function (28), the precise effects of RJ on immune system require further study.

It has been suggested that immunomodulation of a host system could inhibit tumor growth; therefore, immunotherapy is considered as an adjuvant therapy for cancer treatment (29). Certain studies have reported that targeting tumor-associated plasmacytoid dendritic cells to restore their IFN- $\alpha$ production may be an achievable therapeutic strategy to induce antitumor immunity in breast cancer (30-32). These studies suggested that IFN- $\alpha$ was important for the inhibition of breast cancer development (30-32). In the present study, the level of IFN- $\alpha$ in all six groups treated with RJ was significantly increased, indicating that RJ serves an important role in immunomodulation.

The immune stimulating effect of RJ has been investigated. Low concentrations of MEL 174 (final water extract of RJ) and MEL 147 (3-10-dihydroxydecanoic acid) stimulated rat T-cell proliferation in vitro, and resulted in an increased production of IL-2 of Type 1 T helper (Th1)-associated cytokines (33). The results obtained in the present study support the above findings. The serum concentration of IL-2 in PTRJ and TRJ-treated mice $(0.5$ and $1.5 \mathrm{~g} / \mathrm{kg})$ were significantly higher compared with that of the 4T1 control group. For the other groups treated with RJ, the level of IL-2 was slightly; however, not significantly increased. Thus, these results suggest that the IL-2 cytokine is involved in tumor growth inhibition. The levels of IL-4, a Type $2 \mathrm{~T}$ helper (Th2)-associated cytokine, in the PTRJ0.5, PTRJ1.0 and TRJ1.5 groups, and IL-10 in the PTRJ1.0 group were significantly reduced compared with that of the 4T1 control group. Furthermore, the levels of IL-4 and IL-10 were slightly, but insignificantly decreased in all other groups treated with RJ. These data suggest that treatment with RJ suppressed Th2 activity and associated cytokine release in 4T1-bearing mice. The production of IL- 4 may be restrained by major 
royal jelly protein 3 , isolated from RJ major proteins (34). No significant difference was identified among the IFN- $\gamma$ value of the groups in the experiment. However, the level of IFN- $\gamma$ in the PTRJ0.5, PTRJ1.5, and PTRJ0.5 groups was slightly higher compared that of 4T1 control group. Together, these results suggest that the RJ immunoprotective role acts by increasing the level of Th1-associated cytokines and suppressing the level of Th2-associated cytokines. RJ treatment resulted in increased Th1/Th2 cytokine ratios in the present study, and similar results were also reported by Oka et al (35) and Erem et al (36).

It was reported that oxidant stress was important to carcinogenesis and the accumulation of reactive oxygen species (37), and it highlighted the significance of antioxidant defense enzymes for cancer therapy. Certain reports suggested that patients with breast cancer require improved antioxidation abilities $(38,39)$. It was hypothesized that the antitumor effects of RJ may be partially due to its antioxidant properties. Regarding the observations made in the current study, the levels of SOD and T-AOC in the serum, in addition to the level of T-AOC, and GR in the liver were significantly increased following RJ treatment. Furthermore, in the liver, the level of SOD in the TRJ1.5 group was significantly increased compared with that of the 4T1 control group. The concentration of T-AOC and GR in the kidney was significantly increased in the PTRJ0.5 group. Additionally, the levels of T-AOC, GR and SOD in the kidney of the PTRJ1.0, PTRJ1.5, TRJ0.5, and TRJ1.0 groups were augmented, but the increases were not statistically significant. These data suggest that although the functions of RJ on the antioxidant activities in the serum, liver and kidney were different, overall, the antioxidant activities increased following RJ supplementation in 4T1-bearing mice. These results are in agreement with the literature on the antioxidant properties of RJ $(40,41)$. It is possible that administration of RJ enhances antioxidant activity, since RJ contains a mixture of peptides, flavonoids and cinnamic acid derivatives (42). Thus, the antioxidant effect of RJ may contribute to the growth inhibition of 4T1 mammary tumors in mice.

There are other cytokines, besides the cytokines mentioned in the present study, which possess antitumor effects in vivo $(29,43)$. It is possible that the antitumor effect of RJ on 4T1-bearing mice is associated with the action of such cytokines, including, TNF- $\alpha$, IL-1, IL-6, IL-13 (41) and IL-27 (29).

In conclusion, the antioxidant and immunomodulatory effects of RJ on the 4T1 breast cancer mice model were investigated in the present study. RJ treatment was demonstrated to reduce the development of breast tumors in mice, and improve the antioxidant activity in the serum, liver and kidney, particularly following PTRJ treatment. These positive effects corroborate the efficacy of RJ supplementation in diets. The antioxidant and immunomodulatory effects of RJ likely serve an important antitumor role. Notably, it should be taken into account that the therapeutic method of RJ may be used $\geq 14$ days prior to tumor inoculation.

\section{Acknowledgements}

The research was supported by the earmarked fund provided by the Finance Department of Fujian province (grant no. K81MLV02A) and the Modern Agro-industry Technology Research System (grant no. CARS-45-KXJ3).

\section{References}

1. Isidorov V, Czyżewska U, Isidorova A and Bakier S: Gas chromatographic and mass spectrometric characterization of the organic acids extracted from some preparations containing lyophilized royal jelly. J Chromatogr B Analyt Technol Biomed Life Sci 877: 3776-3780, 2009.

2. Fujiwara S, Imai J, Fujiwara M, Yaeshima T, Kawashima T and Kobayashi K: A potent antibacterial protein in royal jelly. purification and determination of the primary structure of royalisin. J Biol Chem 265: 11333-11337, 1990.

3. Karaca T, Uz YH, Demirtas S, Karaboga I and Can G: Protective effect of royal jelly in 2,4,6 trinitrobenzene sulfonic acid-induced colitis in rats. Iran J Basic Med Sci 18: 370-379, 2015.

4. Arzi A, Olapour S, Yaghooti H and Sistani Karampour N: Effect of royal jelly on formalin induced-inflammation in rat hind paw. Jundishapur J Nat Pharm Prod 10: e22466, 2015.

5. Yildirim S, Karadeniz A, Karakoc A, Yildirim A, Kalkan Y and Şimşek N: Effects of royal jelly on liver paraoxonase activity in rats treated with cisplatin. Turk J Med Sci 42: 367-375, 2012.

6. Niu K, Guo H, Guo Y, Ebihara S, Asada M, Ohrui T, Furukawa K, Ichinose M, Yanai K, Kudo Y, et al: Royal jelly prevents the progression of sarcopenia in aged mice in vivo and in vitro. J Gerontol A Biol Sci Med Sci 68: 1482-1492, 2013.

7. Nomura M, Maruo N, Zamami Y, Takatori S, Doi S and Kawasaki H: Effect of long-term treatment with royal jelly on insulin resistance in otsuka long-evans tokushima fatty (OLETF) rats. Yakugaku Zasshi 127: 1877-1882, 2007.

8. Kashima Y, Kanematsu S, Asai S, Kusada M, Watanabe S, Kawashima T, Nakamura T, Shimada M, Goto T and Nagaoka S: Identification of a novel hypocholesterolemic protein, major royal jelly protein 1, derived from royal jelly. PLoS One 9: e105073, 2014 .

9. Nakaya M, Onda H, Sasaki K, Yukiyoshi A, Tachibana H and Yamada K: Effect of royal jelly on bisphenol A-induced proliferation of human breast cancer cells. Biosci Biotechnol Biochem 71: 253-255, 2007.

10. Salazar-Olivo L and Paz-González V: Screening of biological activities present in honeybee (Apis mellifera) royal jelly. Toxicol In Vitro 19: 645-651, 2005.

11. Oršolić N, Terzić S, Šver L and Bašić I: Honey-bee products in prevention and/or therapy of murine transplantable tumours. J Sci Food Agric 85: 363-370, 2005.

12. Šimúth J, Bíliková K, Kováčová E, Kuzmová Z and Schroder W: Immunochemical approach to detection of ddulteration in honey: Physiologically active royal jelly protein stimulating TNF-alpha release is a regular component of honey. J Agric Food Chem 52: 2154-2158, 2004.

13. Mishima S, Suzuki KM, Isohama Y, Kuratsu N, Araki Y, Inoue $M$ and Miyata T: Royal jelly has estrogenic effects in vitro and in vivo. J Ethnopharmacol 101: 215-220, 2005.

14. Suzuki KM, Isohama Y, Maruyama H, Yamada Y, Narita Y, Ohta S, Araki Y, Miyata T and Mishima S: Estrogenic activities of fatty acids and a sterol isolated from royal jelly. Evid Based Complement Alternat Med 5: 295-302, 2008.

15. Althuis MD, Fergenbaum JH, Garcia-Closas M, Brinton LA, Madigan MP and Sherman ME: Etiology of hormone receptor-defined breast cancer: A systematic review of the literature. Cancer Epidemiol Biomarkers Prev 13: 1558-1568, 2004.

16. Samavat $H$ and Kurzer MS: Estrogen metabolism and breast cancer. Cancer Lett 356: 231-243, 2015.

17. Iwase H: Treatment strategy for metastatic breast cancer with estrogen receptor-positive tumor. Int J Clin Oncol 20: 249-252, 2015.

18. Taavoni S, Barkhordari F, Goushegir A and Haghani H: Effect of Royal Jelly on premenstrual syndrome among Iranian medical sciences students: A randomized, triple-blind, placebo-controlled study. Complement Ther Med 22: 601-606, 2014.

19. Pulaski BA and Ostrand-Rosenberg S: Reduction of established spontaneous mammary carcinoma metastases following immunotherapy with major histocompatibility complex class II and B7. 1 cell-based tumor vaccines. Cancer Res 58: 1486-1493, 1998.

20. Ferlay J, Shin HR, Bray F, Forman D, Mathers C and Parkin DM: Estimates of worldwide burden of cancer in 2008: GLOBOCAN 2008. Int J Cancer 127: 2893-2917, 2010.

21. Luo KW, Ko CH, Yue GG, Lee JK, Li KK, Lee M, Li G, Fung KP, Leung PC and Lau CB: Green tea (Camellia sinensis) extract inhibits both the metastasis and osteolytic components of mammary cancer 4T1 lesions in mice. J Nutr Biochem 25: 395-403, 2014. 
22. Bincoletto $\mathrm{C}$, Eberlin S, Figueiredo CA, Luengo MB and Queiroz ML: Effects produced by royal jelly on haematopoiesis: Relation with host resistance against ehrlich ascites tumour challenge. Int Immunopharmacol 5: 679-688, 2005.

23. Tamura T, Fujii A and Kuboyama N: Antitumor effects of royal jelly (RJ). Nihon Yakurigaku Zasshi 89: 73-80, 1987 (In Japanese).

24. Shirzad M, Kordyazdi R, Shahinfard N and Nikokar M. Does royal jelly affect tumor cells?. J HerbMed Pharmacol 2: 45-48, 2013.

25. Townsend GF, Morgan JF and Hazlett B: Activity of 10-hydroxydecenoic acid from royal jelly against experimental leukaemia and ascitic tumours. Nature 183: 1270-1271, 1959.

26. Townsend GF, Morgan JF, Tolnai S, Hazlett B, Morton HJ and Shuel RW: Studies on the in vitro antitumor activity of fatty acids I. 10-hydroxy-2-decenoic acid from royal jelly. Cancer Res 20: 503-510, 1960

27. Male D: Reactions against blood cells and platelets. InL Immunology 4th Edition. Roitt I, Brostoff J and Male D (eds) Mosby, London, pp3-23, 1996.

28. Liu RM, Zhang XJ, Liang GY, Yang YF, Zhong JJ and Xiao JH: Antitumor and antimetastatic activities of chloroform extract of medicinal mushroom cordyceps taii in mouse models. BMC Complement Altern Med 15: 216, 2015

29. Murugaiyan G and Saha B: IL-27 in tumor immunity and immunotherapy. Trends Mol Med 19: 108-116, 2013.

30. Sisirak V, Faget J, Gobert M, Goutagny N, Vey N, Treilleux I, Renaudineau S, Poyet G, Labidi-Galy SI, Goddard-Leon S, et al: Impaired IFN- $\alpha$ production by plasmacytoid dendritic cells favors regulatory T-cell expansion that may contribute to breast cancer progression. Cancer Res 72: 5188-5197, 2012.

31. Sisirak V, Vey N, Goutagny N, Renaudineau S, Malfroy M, Thys S, Treilleux I, Labidi-Galy SI, Bachelot $\mathrm{T}$ and Dezutter-Dambuyant $\mathrm{C}$, et al: Breast cancer-derived transforming growth factor- $\beta$ and tumor necrosis factor- $\alpha$ compromise interferon- $\alpha$ production by tumor-associated plasmacytoid dendritic cells. Int J Cancer 133: 771-778, 2013.

32. Escobar G, Moi D, Ranghetti A, Ozkal-Baydin P, Squadrito ML, Kajaste-Rudnitski A, Bondanza A, Gentner B, De Palma M, Mazzieri R and Naldini L: Genetic engineering of hematopoiesis for targeted IFN- $\alpha$ delivery inhibits breast cancer progression. Sci Transl Med 6: 217ra3, 2014.
33. Gasic S, Vucevic D, Vasilijic S, Antunovic M, Chinou I and Colic M: Evaluation of the immunomodulatory activities of royal jelly components in vitro. Immunopharmacol Immunotoxicol 29: 521-536, 2007.

34. Okamoto I, Taniguchi Y, Kunikata T, Kohno K, Iwaki K, Ikeda M and Kurimoto M: Major royal jelly protein 3 modulates immune responses in vitro and in vivo. Life Sci 73: 2029-2045, 2003.

35. Oka H, Emori Y, Kobayashi N, Hayashi Y and Nomoto K: Suppression of allergic reactions by royal jelly in association with the restoration of macrophage function and the improvement of Th1/Th2 cell responses. Int Immunopharmacol 1: 521-532, 2001.

36. Erem C, Deger O, Ovali E and Barlak Y: The effects of royal jelly on autoimmunity in Graves' disease. Endocrine 30: 175-183, 2006.

37. Cebrian A, Pharoah PD, Ahmed S, Smith PL, Luccarini C, Luben R, Redman K, Munday H, Easton DF, Dunning AM and Ponder BA: Tagging single-nucleotide polymorphisms in antioxidant defense enzymes and susceptibility to breast cancer. Cancer Res 66: 1225-1233, 2006.

38. Şener DE, Gönenç A, Akıncı M and Torun M: Lipid peroxidation and total antioxidant status in patients with breast cancer. Cell Biochem Funct 25: 377-382, 2007.

39. Samy RP, Gopalakrishnakone P and Ignacimuthu S: Anti-tumor promoting potential of luteolin against 7,12-dimethylbenz(a) anthracene-induced mammary tumors in rats. Chem Biol Interact 164: 1-14, 2006.

40. Karadeniz A, Simsek N, Karakus E, Yildirim S, Kara A, Can I, Kisa F, Emre H and Turkeli M: Royal jelly modulates oxidative stress and apoptosis in liver and kidneys of rats treated with cisplatin. Oxid Med Cell Longev 2011: 981793, 2011.

41. Jamnik P, Goranovič D and Raspor P: Antioxidative action of royal jelly in the yeast cell. Exp Gerontol 42: 594-600, 2007.

42. Gómez-Caravaca AM, Gómez-Romero M, Arráez-Román D, Segura-Carretero A and Fernández-Gutiérrez A: Advances in the analysis of phenolic compounds in products derived from bees. J Pharm Biomed Anal 41: 1220-1234, 2006.

43. Da Silva RJ, Da Silva MG, Vilela LC and Fecchio D: Cytokine profile of ehrlich ascites tumor treated with Bothrops jararaca venom. Mediators Inflamm 11: 197-201, 2002. 